\title{
The Fungal community in non-rhizosphere soil of Panax ginseng are driven by different cultivation modes and increased cultivation periods
}

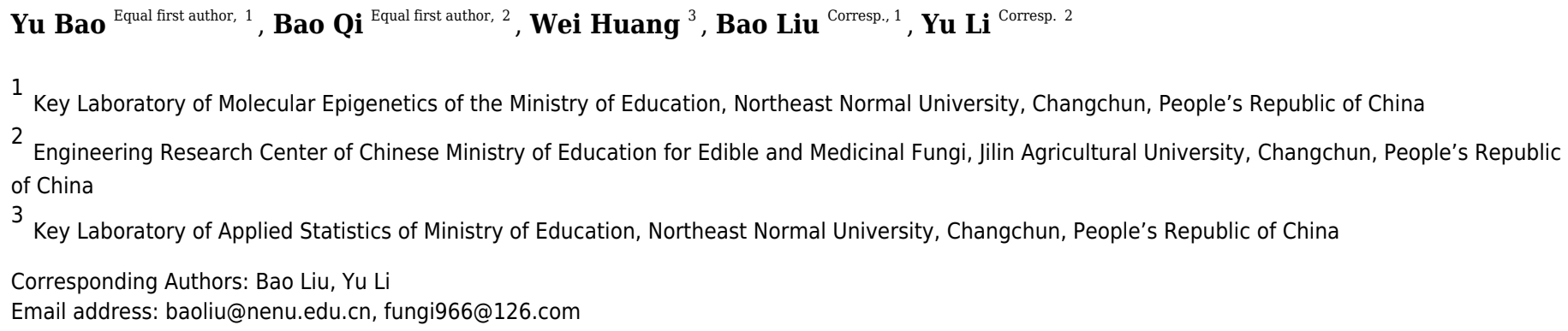

Continuous cropping obstacles severely hindered the sustained development of the ginseng industry. Among the obstacles, an imbalance of soil microbiome community was considered one of the major culprits. Fungal community is an essential part of the soil microbiome community. Extensive characterization of the fungal community composition and variation during ginseng cultivation will help us understand the mechanism underlying continuous cropping obstacles. By using a high-throughput amplicon sequencing method, the non-rhizospheric fungal community of farmland cultivated ginseng of 2-y-old (C2) and 5 -y-old (C5), understory wild ginseng of 15-y-old (W15) and 35-y-old (W35), fallow fields which have been abandoned for 10 (F10) years were characterized. Farmland cultivated ginseng and understory wild ginseng harbored distinct non-rhizospheric fungal communities, and extension of cultivation periods enlarged the fungal community difference between two cultivation modes. Extended cultivation periods significantly decreased the OTU richness and PD whole tree indices, and OTU number and cultivation periods were negatively correlated. Extension of cultivation periods led to an increased abundance of pathotrophs. Still, the increased abundance of pathotrophs may not be the leading cause of severe continuous cropping obstacles in farmland cultivated ginseng. Compared with understory wild ginseng, farmland cultivated ginseng had a lower abundance of symbiotrophs and a higher abundance of saprotrophs. This changed symbiotrophs/saprotrophs ratio may have some correlation with the severe continuous cropping obstacles that occurred in farmland cultivated ginseng. Fallowing on the fungal community of the non-rhizosphere soil was generally opposite of that of extension of ginseng cultivation periods. The impacts of farmland cultivation on the fungal community of the non-rhizosphere soil can last for decades, even if the following is practiced. 


\section{The fungal community in non-rhizosphere soil of 2 Panax ginseng are driven by different cultivation 3 modes and increased cultivation periods}

4

5

6

7

8

Yu Bao ${ }^{*}$, Bao Qi ${ }^{1,2 *}$, Wei Huang ${ }^{3}$, Bao Liu ${ }^{1}$ and $\mathrm{Yu} \mathrm{Li}^{1,2}$

${ }^{1}$ Key Laboratory of Molecular Epigenetics of the Ministry of Education, Northeast Normal University, Changchun 130024, Jilin, People's Republic of China

${ }^{2}$ Engineering Research Center of Chinese Ministry of Education for Edible and Medicinal Fungi, Jilin Agricultural University, Changchun130118, Jilin, People's Republic of China

${ }^{3}$ Key Laboratory of Applied Statistics of Ministry of Education, Northeast Normal University, Changchun 130024, Jilin, People's Republic of China

Corresponding Author:

Bao Liu ${ }^{1}$

Changchun 130024 Jilin, People's Republic of China

Email address: baoliu@nenu.edu.cn

$\mathrm{Yu} \mathrm{Li}{ }^{1,2}$

Changchun, 130118 Jilin, People's Republic of China

Email address: fungi966@126.com

\section{Abstract}

Continuous cropping obstacles severely hindered the sustained development of the ginseng industry. Among the obstacles, an imbalance of soil microbiome community was considered one of the major culprits. Fungal community is an essential part of the soil microbiome community. Extensive characterization of the fungal community composition and variation during ginseng cultivation will help us understand the mechanism underlying continuous cropping obstacles. By using a high-throughput amplicon sequencing method, the non-rhizospheric fungal community of farmland cultivated ginseng of 2-y-old (C2) and 5-y-old (C5), understory wild ginseng of 15y-old (W15) and 35-y-old (W35), fallow fields which have been abandoned for 10 (F10) years were characterized. Farmland cultivated ginseng and understory wild ginseng harbored distinct non-rhizospheric fungal communities, and extension of cultivation periods enlarged the fungal community difference between two cultivation modes. Extended cultivation periods significantly decreased the OTU richness and PD whole tree indices, and OTU number and cultivation periods were negatively correlated. Extension of cultivation periods led to an increased abundance of pathotrophs. Still, the increased abundance of pathotrophs may not be the leading cause of severe continuous cropping obstacles in farmland cultivated ginseng. Compared with understory wild ginseng, farmland cultivated ginseng had a lower abundance of symbiotrophs and a higher abundance of saprotrophs. This changed symbiotrophs/saprotrophs ratio may have some 
40 correlation with the severe continuous cropping obstacles that occurred in farmland cultivated

41 ginseng. Fallowing on the fungal community of the non-rhizosphere soil was generally opposite

42 of that of extension of ginseng cultivation periods. The impacts of farmland cultivation on the

43 fungal community of the non-rhizosphere soil can last for decades, even if the following is

44 practiced.

45

46

47

48

49

50

51

52

53

54

55

56

57

58

59

60

61

62

63

64

65

66

67

68

69

70

71

72

73

74

75

76

77

78

\section{Introduction}

Ginseng (Panax ginseng C.A. Mey.) has been used as traditional Chinese herbal medicine for thousands of years. Nowadays, the global market value of ginseng is about 3.5 billion US dollars annually, and the demand for ginseng products is still rising. Cultivated ginseng provides the majority of the market demand, which primarily includes two cultivation modes, farmland cultivation (cultivated ginseng) and understory cultivation (wild ginseng). One of the most severe hindrances of ginseng cultivation is continuous cropping obstacles. Ginseng cannot be consecutively cultivated in the same field without causing severe loss of yield and quality. It has been reported that the replantation of ginseng may cause up to $75 \%$ of ginseng seedling death (Wu et al., 2008). Usually, more than 30 periods of crop rotation were needed for successful replanting (Yang et al., 2004), which makes proper soils suitable for ginseng cultivation a scarce resource. Farmland cultivation of ginseng has led to large-scale deforestation in the northeast region of China, which is unsustainable and has severely hindered the sustained development of the ginseng industry.

Several factors have been shown or implicated to correlate with continuous cropping obstacles, one of which is an imbalance of soil microbiome community (Ogweno and $\mathrm{Yu}, 2006$; $\mathrm{Wu}$, 2008). Being an essential part of the soil microbiome community, soil fungal community changes during ginseng cultivation had received much attention, and it has been shown that rhizospheric fungi communities can be affected by different cultivation ages, growth stages and cultivation modes (Li et al., 2012)(Liu, 2013; Xiao et al., 2016; Dong et al., 2018). However, these studies mainly focused on the rhizosphere compartment of soil, which refers to the narrow zone surrounding the root (Estabrook and Yoder, 1998). For most of the soil, namely the nonrhizosphere compartment of soil, little work has been done (Li et al., 2012)(Liu, 2013). Microbial community in ginseng non-rhizosphere and rhizosphere soil were reported to be different, and with the increase of cultivation ages, this difference became more significant ( $\mathrm{Li}$ et al., 2012)(Liu, 2013). However, it is not clear whether the microbial communities in nonrhizosphere soil can also be affected by ginseng cultivation. Meanwhile, due to the technical short come of Random Amplified Polymorphic DNA (RAPD) and Denaturing gradient gel electrophoresis (DGGE), these two studies did not provide many details of the fungi communities dynamics in non-rhizosphere soil (Li et al., 2012)(Liu, 2013). More extensive characterization of the non-rhizospheric fungal community composition and variation during ginseng cultivation is essential to understand the underlying mechanism of continuous cropping obstacles. 
79 In contrast to farmland cultivated ginseng, understory wild ginseng was grown up in the natural

80 forest. It can be consecutively cultivated in the same forest for a long time without causing

81 severe continuous cropping obstacles. Illustrating the fungal community difference between

82 understory wild ginseng and farmland cultivated ginseng will provide insights into the

83 mechanism underlying continuous cropping obstacles. Land fallow is a practical approach to

84 achieve replantation, but the $30-40$ periods of rotation time needed is too long. Extensive

85 characterization of the non-rhizospheric fungal community dynamics during land fallow will

86 help us understand the mechanisms underlying crop rotation and provide clues to accelerate this

87 process.

88 The present study was designed to characterization the influence of different cultivation ages and

89 cultivation modes to the non-rhizospheric fungal community of ginseng, hope to provide insights

90 into the mechanism underlying continuous cropping obstacles.

91

92 Materials \& Methods

93 Soil sample collection and DNA extraction

94 Soil samples were collected in growth season (July 10 ${ }^{\text {th }}$ 2016) from Dadong Village (42.31 N

95 127.19 E), Fusong, Jilin Province of China. Fusong is the central ginseng production region of

96 China, and the Dadong Village is one of the several places where understory wild ginseng has

97 been grown for over 40 years. Soil samples were collected from fields of farmland cultivated

98 ginseng of 2-y-old (C2) and 5-y-old (C5), understory wild ginseng of 15-y-old (W15), and 35-y-

99 old (W35), fallow fields which have been abandoned for 10 (F10) years after five years of

100 ginseng farmland cultivation. We chose 2-y-old and 5-y-old farmland cultivated ginseng because

101 after two years of growth, disease occurrence and death rates of farmland cultivated ginseng

102 generally increase (Dong et al., 2018) and farmland cultivated ginseng is usually harvested after

103 five years of farmland cultivation. Thirty-five-y-old understory wild ginseng was the oldest

104 understory wild ginseng we can found. Coupled with the 15-y-old understory wild ginseng, we

105 can investigate the long-term influence of understory wild ginseng cultivation on the non-

106 rhizospheric fungal community.

107 The understory wild ginseng used in this study was direct planted to the natural forest and grown

108 without any manual intervention. The soil used for farmland cultivation was removed from the

109 same area of natural forest where understory wild ginseng was grown. Fallow fields used in this

110 study have been abandoned for ten years without planting any crops to avoid the influence of

111 crop cultivation practice on soil microbial community.

112 Non-rhizosphere soil components of ginseng (20 cm away from ginseng plants) were collected

113 as study materials with three biological replications. For each sample, 40 soil cores were

114 collected from about $2500 \mathrm{~m}^{2}$ circular plots and pooled together. Each soil core was collected

115 using a $5 \mathrm{~cm}$ diam PVC tube, which was hammered into the soil and collect the soil from the

116 surface to down to $5 \mathrm{~cm}$ depth (Tedersoo et al., 2014). Power Soil DNA Isolation kit (MoBio,

117 Carlsbad, CA USA) was used to extracted DNA from about $10 \mathrm{~g}$ soil according to the

118 manufacturer instructions.

Peer] reviewing PDF | (2019:05:37672:1:1:NEW 24 Jun 2020) 


\section{Illumina sequencing of ITS rRNA gene amplicons}

121 Fungi intergenic transcribed sequence (ITS) universal primers F2045 (5'-

122 GCATCGATGAAGAACGCAGC-3') and R2390 (5'- TCCTCCGCTTATTGATATGC-3) were used. Library construction and sequencing were carried out by

124

125

126

127

128

129

130

131

132

133

134

135

136

137

138

139

140

141

142

143

144

145

146

147

148

149

150

151

152

153

154

155

156

157

158

Realbio Genomics Institute (Shanghai, China). Illumina Hiseq 2500 platform was used to perform amplicon sequencing and generated about 100100,000 $250 \mathrm{bp}$ paired-end (PE) reads in each sample. Original data generated in this study have been deposited into the NCBI SRA database, and the accession number was PRJNA523683.

\section{Data analyses}

PANDAseq (Masella et al., 2012) was used to assemble the paired-end (PE) reads, quality assessment and filtering were carried out with QIIME1 pipeline with default parameters (Caporaso et al., 2010). Chimeric sequences were identified and filtered by the UCHIME program (Edgar et al., 2011). USEARCH implemented in QIIME1 software (Sachs et al., 2011) was utilized to assign Operational taxonomic units (OTU) at the 97\% sequence identity level. The most abundant sequence of each OUT was chosen as the representative sequence and then taxonomically classified using the RDP classifier against the Ribosomal Database Project (RDP) database (Wang et al., 2007, Brown et al. 2013). OTUs which belong to mitochondrion or chloroplast and singleton OTUs were removed. Alpha and beta diversities were calculated using the QIIME1 pipeline (Caporaso et al. 2010) after rarefied to 132764 sequences for each sample. We used linear discriminant analysis effect size (LEfSe) (Segata et al. 2011) to identify the differentiation of fungal communities between different samples at multiple taxonomical levels, Kruskal-Wallis test $P<0.05$ and LDA score of $>2$ were used as thresholds. Principal coordinate analyses (PCoA) based on unweighted UniFrac distance metrics were carried out using the QIIME1 pipeline (Caporaso et al. 2010). ADONIS was carried out using the $\mathrm{R}$ package vegan (Dixon, 2003).

Differences of alpha diversity indexes between samples were determined by Tukey's method (multiple comparisons of means), and normality and homogeneity of variance analysis were respectively performed by using the Shapiro-Wilk normality test and Brarlett test. These analyses were carried out using R software (Version 3.5.2, https://www.r-project.org/). OTUs were parsed against the FUNGuild database to assign putative trophic strategies (Nguyen et al., 2016).

\section{Regression analysis}

We used a linear model (LM) regression analysis to test the relationship between OTU richness and cultivation periods. Standardized major axis (SMA) analysis was carried out using (S)MATR package in R (Falster et al., 2006; Warton et al., 2011) to fit a line of best fit and to obtain a slope estimate and R2 value.

Peer] reviewing PDF | (2019:05:37672:1:1:NEW 24 Jun 2020) 
159 Results

160 Variation in fungi community compositions

161 Soil samples included four cultivation periods: 2-y-old (C2) and 5-y-old (C5) cultivated ginseng, 162 15-y-old (W15), and 35-y-old (W35) understory wild ginseng. Intergenic transcribed sequence 163 (ITS) gene amplicon sequencing generated 135,340-189,257 reads and was rarefied to 132,764 164 clean reads for each sample. The Good's coverage scores ranged from 0.996792 to 0.999862 , 165 indicating that the sequencing depth was sufficient to quantify the fungi communities (Table 1). 166 According to Principal Co-ordinates Analysis (PCoAs) analyses, samples from farmland 167 cultivated ginseng and understory wild ginseng showed clear separation (Figure 1A). PCoAs1 168 and PCoAs2 explained $23.13 \%$ and $16.68 \%$ of variation, respectively (Figure 1A). ADONIS 169 analysis showed that fungi communities in non-rhizosphere soil of farmland cultivated ginseng 170 and understory wild ginseng were significantly different $\left(\mathrm{R}^{2}=0.22377, P=0.003\right)$. Only $27.4 \%$ 171 of OTUs from farmland cultivated ginseng and understory wild ginseng were overlapped (1494 172 out of 5446) (Figure 1B). There was 1310 common OTUs (28.1\% of a total of 4663 OTUs in C2 173 and W15) between $\mathrm{C} 2$ and W15, the number of common OTUs between C5 and W35 decreased 174 to 285 (11.8\% of a total of 2414 OTUs in C5 and W35, Figure 1B). These results indicated that with the extension of cultivation periods, the differences of fungal community between the two cultivation modes were enlarged. In both farmland cultivated ginseng and understory wild ginseng, Ascomycota and Basidiomycota were dominant phyla, which accounted for 75.1\%91.1\% of the total OTUs (Supplemental file 1). LEfSe analysis showed that Agaricomycetes,

180

181

182

183

184

185

186

187

188

189

190

191

192

193

194

195

196

197

Pezizomycetes and Glomeromycota were significantly enriched in understory wild ginseng compared to that of cultivated ginseng. In contrast, Sordariomycetes, Dothideomycetes, Ascomycota_unidentified, Eurotiomycetes, Tremellomycetes, Lecanoromycetes, Cystobasidiomycetes, and Wallemiomycetes were significantly enriched in farmland cultivated ginseng compared to that of understory wild ginseng (Figure1C, D, LEfSe, $P<0.05, \mathrm{LDA}>2$ ).

Extension of cultivation periods lead to decreased OTU richness and PD whole tree indices in both cultivation modes

Extension of cultivation periods significantly decreased the OTU richness and PD whole tree indices in both understory wild ginseng and farmland cultivated ginseng (Tukey's method, $P<$ 0.01; Table 1). After another three years of farmland cultivation, the OTU number decreased from 3261 OTUs in C2 to 1724 in C5, which was $52.9 \%$ of that in C2 (5914 OTUs) (Figure 1B, Supplemental file 1). Similar results were also found in understory wild ginseng, coupled with the extension of cultivation periods, the OTU number decreased from 2712 in W15 to 975 in W35 (Figure 1B, Supplemental file 1). The OTU number and cultivation periods were negatively correlated (SMA, p-value $=0.0020962, \mathrm{R} 2=0.62863$, slope $=-48.42971,95 \%$ CI's $=-73.50041$ to -31.91053 , Figure 2). However, the species evenness was not affected by the extension of cultivation periods in both cultivation modes, as indicated by Simpson indices (Tukey's method, $P>0.05$, Table 1$)$.

PeerJ reviewing PDF | (2019:05:37672:1:1:NEW 24 Jun 2020) 
198

199

200

201

202

203

204

205

206

207

208

209

210

211

212

213

214

215

216

217

218

219

220

221

222

223

224

225

226

227

228

229

230

231

232

233

234

235

236

237

With the extension of cultivation periods, non-rhizosphere soil of more extended cultivation periods lost relatively the same amount of OTUs in the two cultivation modes (2000 OTU loss in W35 and 2241 loss in C5), while non-rhizosphere soil of farmland cultivated ginseng acquired more OTUs than that in understory wild ginseng (704 OUT gain in C5 compared with 263 in W35, Figure 1B). Only a small proportion of the lost or gained OTUs in more extended cultivation periods of the two cultivation modes were overlapped (Figure 1B). Specifically, there was 531 common OUT loss (26.6\% in W35 and $23.7 \%$ in C5) in samples of more extended cultivation periods, and the number of common OUT gain in samples of more extended cultivation period was 19 (7.2\% in W35 and $2.7 \%$ in C5, Figure 1B). Under farmland cultivation mode, with the extension of cultivation periods the abundance of Leotiomycetes, Ascomycota_unidentified, Microbotryomycetes, and Agaricostilbomycetes were significantly increased, while the abundance of Agaricomycetes, Incertae_sedis_10, Pezizomycetes, Chytridiomycetes, Orbiliomycetes, Incertae_sedis_14, Glomeromycetes, and Saccharomycetes were significantly decreased (Figure 3, LEfSe, $P<0.05$, LDA $>2$ ). Under understory wild ginseng cultivation mode, with the extension of cultivation periods, the abundance of Incertae_sedis_14 was significantly increased, and the abundance of Chytridiomycetes_unidentified was significantly decreased in non-rhizosphere soil compartment (Figure 4, LefSe, $P<0.05$, LDA $>2$ ).

\section{Influence of fallowing on non-rhizospheric fungal community}

Ginseng plants were harvested after five years of farmland cultivation, and the resulted fields were abandoned for ten years without growing any crops. According to PCoAs analyses, samples from fallow soil and farmland cultivated ginseng showed clear separation (Figure 1A). ADONIS analysis showed that non-rhizospheric fungi communities in the fallow field and farmland cultivated ginseng were significantly different $\left(\mathrm{R}^{2}=0.35168, P=0.009\right)$. After fallowing for ten years, the OTU richness, PD whole tree indices, and species evenness in non-rhizosphere soils were significantly increased (Tukey's method, $P<0.05$; Table 1). The OTU richness in F 10 recovered to 2969 OTUs, but still less than that of C2 (Figure 5A, Supplemental file 1). Fallowing recovered 31.3\% (702 out of 2241) of the lost OTUs in C5 during the extension of cultivation periods, and for the gained OTUs, $38.2 \%$ (274 out of 704) of which were conserved in F10 (Figure 5A). The other 1293 OTUs were gained OTUs in F10 (Figure 5A). These results demonstrated that an increase of OTU richness in fallowed soil was achieved mainly by the gain of new OTUs other than by recovering the lost OTUs during the extension of cultivation periods. Compared with C5, the abundance of Pezizomycetes, Chytridiomycetes, Pucciniomycetes, Chytridiomycetes_unidentified, Orbiliomycetes, Incertae_sedis_14, and Glomeromycetes were significantly increased, and the abundance of Eurotiomycetes, Ascomycota_unidentified, Lecanoromycetes, Cystobasidiomycetes, and Agaricostilbomycetes were significantly decreased in F10 (Figure 5B, LefSe, $P<0.05, \mathrm{LDA}>2$ ).

\section{Trophic mode prediction}


238 FUNGuild software was used to assign a putative life strategy. Compared with understory wild

239

240

241

242

243

244

245

246

247

248

249

250

251

252

253

254

255

256

257

258

259

260

261

262

263

264

265

266

267

268

269

270

271

272

273

274

275

276

277

ginseng, the non-rhizosphere soil of farmland cultivated ginseng had a lower abundance of symbiotrophs (Figure 6). What is more, with the extension of the cultivation period, the abundance of symbiotrophs became even lower (Figure 6). After fallowing for ten years, the abundance of symbiotrophs in F10 recovered and became slightly higher than C2 (31.02\% in F10 vs. $29.37 \%$ in C2). In contrast, the non-rhizosphere soil of farmland cultivated ginseng had a higher abundance of saprotrophs than that of understory wild ginseng (Figure 6). Moreover, with the extension of the cultivation period, the abundance of saprotrophs became even higher (Figure 6). Similar to the result of symbiotrophs, the abundance of saprotrophs in F10 became even lower than $\mathrm{C} 2$ (42.27\% in F10 vs. $56.49 \%$ in C2). Extension of cultivation period leads to the increased abundance of pathotrophs in both understory wild ginseng and farmland cultivated ginseng. F10 has the highest abundance of pathotrophs.

\section{Discussion}

Although the soil used to grow farmland cultivated ginseng was removed from the same forest where understory wild ginseng was grown, fungi communities in non-rhizosphere soil of farmland cultivated ginseng were significantly different from that of understory wild ginseng (ADONIS analysis, $\mathrm{R}^{2}=0.22377, P=0.003$ ). Only $27.4 \%$ ( 1494 out of 5446 ) of OTUs between farmland cultivated ginseng and understory wild ginseng were overlapped (Figure 1B). In previous studies, it had been shown that rhizospheric fungi communities are affected by different ginseng cultivation modes (Li et al., 2012)(Liu, 2013; Xiao et al., 2016; Dong et al., 2018). The data presented here proved that fungi communities of non-rhizosphere soil could also be affected by different cultivation modes and different cultivation ages, which means that ginseng can affect a significant part of nearby soil other than just influence the narrow zone tightly surrounding the root namely rhizosphere.

In both understory wild ginseng and farmland cultivated ginseng, extended cultivation periods significantly decreased the OTU richness and PD whole tree indexes of non-rhizosphere soil, and the OTU number and cultivation period were negatively correlated (SMA $P<0.05$ ). Although being different compartments of soil and containing significantly different fungal diversity index (Liu, 2013), similar results have been reported in rhizosphere soil of farmland cultivated ginseng in the vegetative stage (Dong et al., 2018). In Panax notoginseng, a similar result in rhizosphere soil has also been reported (Dong et al., 2016). Farmland cultivated ginseng cannot be consecutively cultivated in the same field without causing severe loss of yield and quality. In contrast, understory wild ginseng can be consecutively cultivated in the same forest. The $35-y-$ old (W35) understory wild ginseng had the lowest OUT number and PD whole tree index of all samples. This fact implied that fungal community composition changes rather than decreased fungal diversity might underly the different responses of the two cultivation modes when facing continuous cropping. Despite having the lowest alpha diversity, the non-rhizosphere fungal community of 35-y-old (W35) understory wild ginseng may still maintain some kind of balance, while in farmland cultivated ginseng, this balance may have been interrupted.

Peer) reviewing PDF | (2019:05:37672:1:1:NEW 24 Jun 2020) 
278 Coupled with the decreased fungi diversity, extension of cultivation periods led to an increased

279 abundance of pathotrophs in both understory wild ginseng and farmland cultivated ginseng

280 (Figure 3). This result resembled the previous reports that there is a positive correlation between

281 fungal diversity and the suppression of soil-borne plant pathogens (NITTA, 1991)(Garbeva et al.,

282 2006). However, the increased abundance of pathotrophs in farmland cultivated ginseng may not

283 be the leading cause of severe continuous cropping obstacles in farmland cultivation ginseng,

284 because understory wild ginseng had an even higher abundance of pathotrophs than farmland

285 cultivated ginseng.

286 Compared with understory wild ginseng, farmland cultivated ginseng had a lower abundance of

287 symbiotrophs, and with the extension of cultivation periods, the abundance of symbiotrophs

288 became even lower (Figure 3). Meanwhile, compared with understory wild ginseng, farmland

289 cultivated ginseng had a higher abundance of saprotrophs, and with the extension of cultivation

290 periods, the abundance of saprotrophs became even higher (Figure 3). This changed

291 symbiotrophs/saprotrophs ratio may have some correlation with the severe continuous cropping

292 obstacles that occurred in farmland cultivated ginseng compared with understory wild ginseng.

293 The effects of fallowing on the fungal community of the non-rhizosphere soil were generally

294 opposite to that of extension of ginseng cultivation periods. Fallowing significantly changed the

295 fungal community composition but in the opposite direction compared to that of extension of

296 ginseng cultivation periods. Extension of cultivation periods significantly increased the

297 abundance of Agaricostilbomycetes and Ascomycota_unidentified, while significantly decreased

298 the abundance of Pezizomycetes, Chytridiomycetes, Orbiliomycetes, Incertae_sedis_14, and

299 Glomeromycetes. In contrast, compared with C5, the abundance of Agaricostilbomycetes and

300 Ascomycota_unidentified was significantly decreased, and the abundance of Pezizomycetes,

301 Chytridiomycetes, Orbiliomycetes, Incertae_sedis_14, and Glomeromycetes were significantly

302 increased in fallowed soil (F10). Fallowing significantly increased the fungal OTU richness and

303 PD whole tree indices, which were also opposed to that of extension cultivation periods. Similar

304 results have been reported in studies carried out on bacteria microbiome (Liu et al., 1983; Li et

305 al., 2011).

306 It should be noted that the overall OTU number in F10 was still less than that in C2, and the 307 increase of OTU richness was achieved mainly by the gain of new OTUs (1293 OTUs) other 308 than recovering the lost OTUs (702 OTUs) during the extension of cultivation period. After ten 309 years of soil fallowing, $38.9 \%$ (274 out of 704) newly emerged OTUs in C5 relative to C2 were 310 still existed in F10, and only $31.3 \%$ (702 out of 2241) of the lost OTUs in C5 relative to C2 311 reappeared in F10. These results indicate that the effects of ginseng farmland cultivation on the 312 fungal community of the non-rhizosphere soil can last for decades, and ten years of fallow only 313 partly restored the fungal community changes caused by farmland cultivation. This result is 314 consistent with the farmer's experience that usually 30-40 years of crop rotation was needed to 315 achieve successful replantation (Yang et al., 2004).

316

\section{Conclusions}


318 Non-rhizosphere soil of farmland cultivated ginseng and understory wild ginseng harbored

319 distinct fungal communities, and with the increase of cultivation periods, these differences were

320 enlarged. In both understory wild ginseng and farmland cultivated ginseng, the extension of

321 cultivation periods significantly decreased the OTU richness and PD whole tree indices.

322 Extension of cultivation periods led to an increased abundance of pathotrophs, but the increased

323 abundance of pathotrophs may not be the leading cause of severe continuous cropping obstacles

324 in farmland cultivated ginseng. Compared with understory wild ginseng, farmland cultivated

325 ginseng had a lower abundance of symbiotrophs and a higher abundance of saprotrophs. This

326 changed symbiotrophs/saprotrophs ratio may have some correlation with the severe continuous

327 cropping obstacles that occurred in farmland cultivated ginseng. The effect of fallowing on the

328 fungal community of the non-rhizosphere soil was generally opposite to that of extension of

329 ginseng cultivation periods. The effects of farmland cultivation on the fungal community of the

330 non-rhizosphere soil can last for decades, even if the following is practiced.

331

332

\section{Declarations}

333 Ethics approval and consent to participate

334 Not applicable

335 Consent for publication

336 Not applicable

337 Availability of data and material

338 The original Illumina sequencing data generated in this study have been deposited into the NCBI

339 SRA database, and the accession number was PRJNA523683.

340 Competing interests

341 The authors declare that they have no competing interests.

342 Funding

343 This work was supported by University S\&T Innovation Platform of Jilin Province for Economic

344 Fungi (no. 2014B-1) and Development Plan Project of Jilin Provincial Science and Technology

345 Department 20160520105JH.

346 Authors' contributions

$347 \mathrm{Yu} \mathrm{Li}$, Bao Qi and Bao Liu designed the research. Yu Bao, Bao Qi, Wei Huang performed the

348 experiments. Yu Bao, Bao Qi and Bao Liu analyzed the data. Yu Bao, Bao Qi, Bao Liu and Yu

349 Li wrote the article with input and approval from all authors.

350

351

\section{Acknowledgements}

352 The authors would like to thank Shihua Chen for her assistance in the soil sampling.

353 References

354 Brown CT, Wang Q, Tiedje JM, Fish JA, Cole JR, McGarrell DM, Porras-Alfaro AS, Yanni K, 355 Cheryl R, Chai B. 2013. Ribosomal Database Project: data and tools for high throughput rRNA 356 analysis. Nucleic Acids Research 42:633-642 DOI: 10.1093/nar/gkt1244. 
357 Caporaso JG, Kuczynski J, Stombaugh J, Bittinger K, Bushman FD, Costello EK, Fierer N, Peña

358

359

360

361

362

363

364

365

366

367

368

369

370

371

372

373

374

375

376

377

378

379

380

381

382

383

384

385

386

387

388

389

390

391

392

393

394

AG, Goodrich JK, Gordon JI, Huttley GA, Kelley ST, Knights D, Koenig JE, Ley RE, Lozupone CA, McDonald D, Muegge BD, Pirrung M, Reeder J, Sevinsky JR, Turnbaugh PJ, Walters WA, Widmann J, Yatsunenko T, Zaneveld J, Knight R. 2010. QIIME allows analysis of highthroughput community sequencing data. Nature Methods 7:335-336 DOI:

10.1038/nmeth.f.303.QIIME.

Chen X, Sun X, Bi S, Lv G. 2010. Study on fungi diversity of ginseng rhizosphere soil in northeastern China. Journal of Anhui Agricultural Sciences 38: 5515-5517.

Dong LL, Niu WH, Wang R, Xu J, Zhang L, Zhang J, Chen SL. 2017. Changes of diversity and composition of fungal communities in rhizosphere of Panax ginseng. Zhongguo Zhong Yao Za Zhi 42:443-449 DOI: 10.19540/j.cnki.cjcmm.20161222.032

Dong L, Xu J, Zhang L, Cheng R, Wei G, Su H, Yang J, Qian J, Xu R, Chen S. 2018.

Rhizospheric microbial communities are driven by Panax ginseng at different growth stages and biocontrol bacteria alleviates replanting mortality. Acta Pharmaceutica Sinica B 8:272-282 DOI: 10.1016/j.apsb.2017.12.011.

Edgar RC, Haas BJ, Clemente JC, Quince C, Knight R. 2011. UCHIME improves sensitivity and speed of chimera detection. Bioinformatics 27:2194-2200 DOI: 10.1093/bioinformatics/btr381. Estabrook EM, Yoder JI. 1998. Plant-plant communications: rhizosphere signaling between parasitic angiosperms and their hosts. Plant Physiology 116: 1-7 DOI: 10.1104/pp.116.1.1.

Falster DS, Warton DI, Wright IJ. 2006. SMATR: Standardised major axis tests and routines, ver 2.0. Available at http://www.bio.mq.edu.au/ecology/SMATR/.

Ogweno JO, Yu JQ. 2006. Autotoxic potential in soil sickness: a re-examination. Allelopathy Journal 18:93-101.

Li Y, YingY, Zhao D, Ding W. 2012. Microbial community diversity analysis of Panax ginseng rhizosphere and non-rhizosphere soil using randomly amplified polymorphic DNA method.

Open J. Genet 2: 95-102 DOI: 10.4236/ojgen.2012.22014.

Liu Q. 2013. Study on rhizosphere soil fungal diversity and dynamic change of communities of ginseng cropping. Thesis, Yanbian University.

Masella AP, Bartram AK, Truszkowski JM, Brown DG, Neufeld JD. 2012. PANDAseq :

PAired-eND Assembler for Illumina sequences. BMC Bioinformatics 13:1-7.

Mey CA. 2008. A review on studies of the reason and control methods of succession cropping obstacle of Panax ginseng. Special Wild Economic Animal. Plant Reserch 2 : 68-72.

Wang Q, Garrity GM, Tiedje JM, Cole JR. 2007. Naive Bayesian classifier for rapid assignment of rRNA sequences into the new bacterial taxonomy. Applied and Environmental Microbiology 73: 5261-5267.

Sachs JL, Skophammer RG, Regus JU. 2011. Evolutionary transitions in bacterial symbiosis.

Proceedings of the National Academy of Sciences of the United States of America 108: 1080010807 DOI: $10.1073 /$ pnas.1100304108.

Peer) reviewing PDF | (2019:05:37672:1:1:NEW 24 Jun 2020) 
395 Segata N, Izard J, Waldron L, Gevers D, Miropolsky L, Garrett WS, Huttenhower C. 2011.

396 Metagenomic biomarker discovery and explanation. Genome Biology 12: 1-18 DOI: 10.1186/gb-

397 2011-12-6-r60.

398 Liu SC, Liu MJ, Lu FY, Xu GH. 1983. Effect on the ecological distribution of soil

399 microorganisms and activities of soil enzymes by planting ginseng. Acta Ecologica Sinica 3: 2940034.

401 Tedersoo L, Tedersoo L, Bahram M, Põlme S, Kõljalg U, Yorou NS, Wijesundera R, Ruiz LV, 402 Vasco-palacios AM, Thu PQ, Suija A, Smith ME, Sharp C, Saluveer E, Saitta A, Rosas M, Riit 403 T, Ratkowsky D, Pritsch K, Põldmaa K, Otsing E, Nouhra E, Njouonkou AL, Nilsson RH, 404 Morgado LN, Mayor J, May TW, Majuakim L, Lodge DJ, Lee SS, Larsson K, Kohout P, Hosaka 405 K, Hiiesalu I, Henkel TW, Harend H, Guo L, Greslebin A, Grelet G, Geml J, Gates G, Dunstan 406 W, Dunk C, Drenkhan R, Dearnaley J, Kesel AD, Dang T, Chen X, Buegger F, Brearley FQ. 407 2014. Global diversity and geography of soil fungi global diversity and geography of soil fungi. 408 Science 346:1256688 DOI: 10.1126/science.1256688.

409 Warton DI, Duursma RA, Falster DS, Taskinen S. 2011. SMATR 3 an R package for estimation 410 and inference about allometric lines. Methods in Ecology and Evolution $3: 257-259$.

$411 \mathrm{Wu}$ LJ, Zhao YH, Guan YM, Peng SF. 2008. A review on studies of the reason and control 412 methods of succession cropping obstacle of Panax ginseng C.A. Mey. Special Wild Economic 413 Animal Plant Reserch $2: 68-72$.

414 Xiao C, Yang L, Zhang L, Liu C, Han M. 2016. Effects of cultivation ages and modes on 415 microbial diversity in the rhizosphere soil of panax ginseng. Journal of Ginseng Reserch 40: 2841637 DOI: $10.1016 /$ j.jgr.2015.04.004.

417 Li XY, Jin HQ, Jia B. 2011. Biodiversity of soil microorganisms in the fields of different 418 growing years of Ginseng. Journal of Agricuhural Science Yanbian University 33: 133-136.

419 Yang LM, Chen CB, Wang XQ, Zhang LX, Tian YX. 2004. Ecological restoration and reused 420 modes of old ginseng land in the Changbai mountainous area and its existing problems. Journal 421 of Jilin Agricutural University 26: 546-549, 553.

422

423 Supplemental material

424 Supplemental file 1: Abundance and the taxonomic classification of the fungal OTUs. 425

426 


\section{Figure 1}

Non-rhizosphere soil associated fungal community

(A) distances show separation of Principal coordinate analysis (PCOA) plot using Unweighted UniFrac method. (B) Venn diagrams illustrating the number of those OTUs that are common between farmland cultivated ginseng and understory wild ginseng samples. (C) Taxonomic classification and relative abundance of fungal community of each sample at the phylum level. (D) LEfSe comparison of fungi community between non-rhizosphere soil of farmland cultivated ginseng and understory wild ginseng. Cladogram represented the hierarchical structure of differently abundant taxa. The taxonomic levels from phylum to family are labelled. 
A

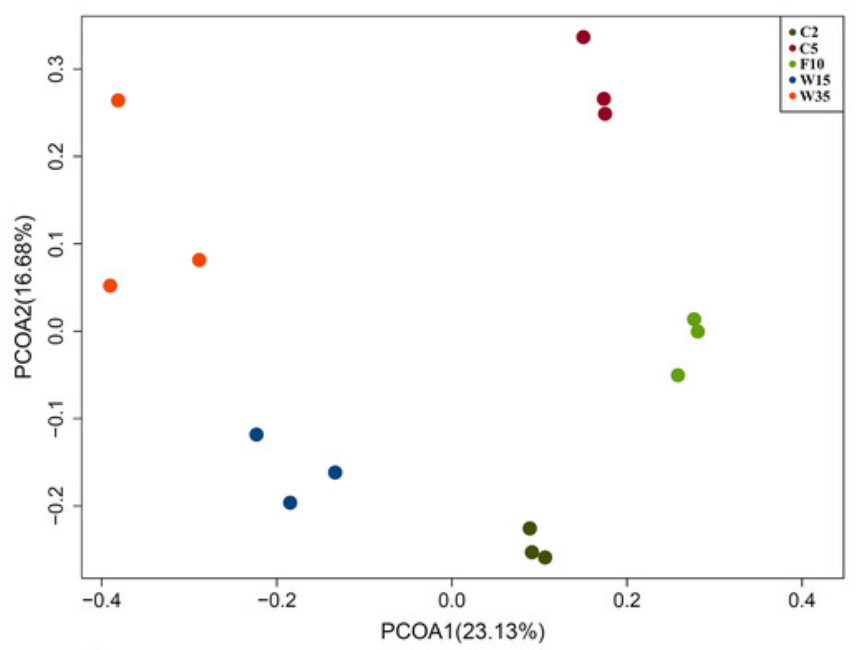

B

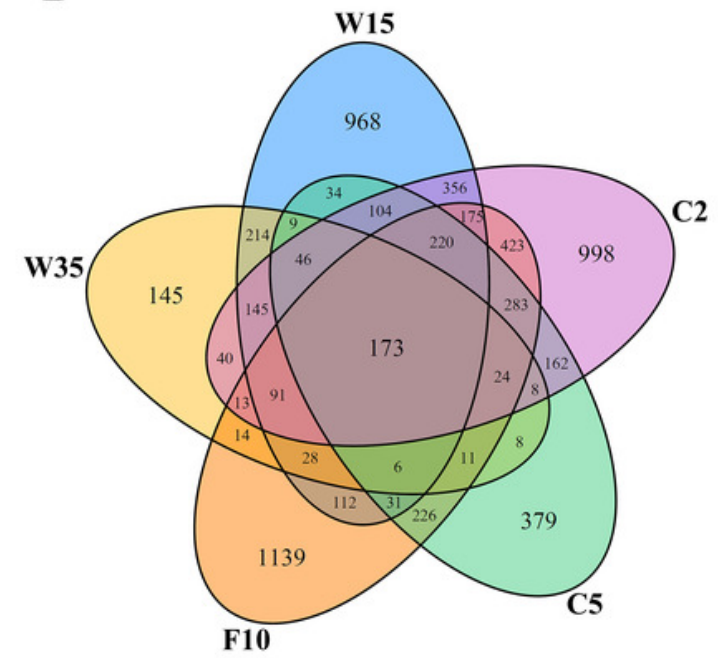

D
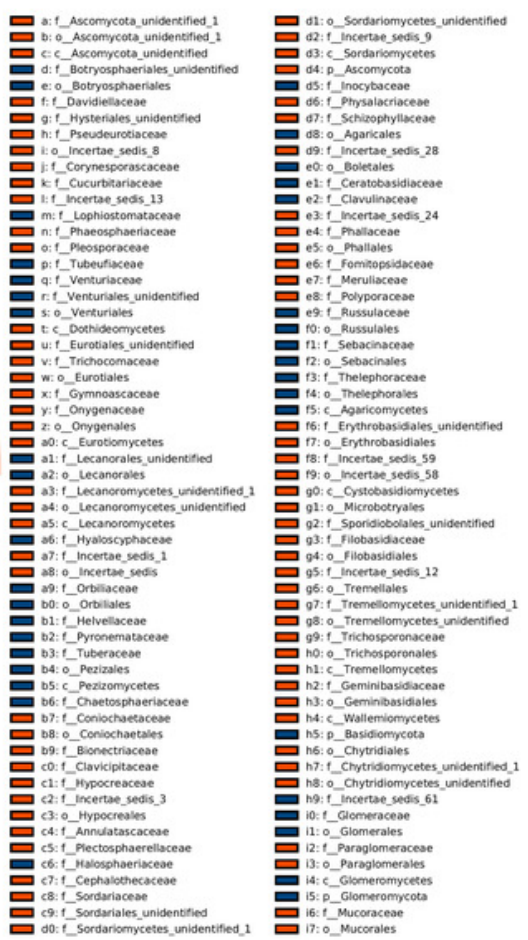
Figure 2

Regression analyses showing the negative relationship between OTU number and cultivation period

The correlation was statistically significant (SMA, $p$-value $=0.0020962, R 2=0.62863$, slope $=-48.42971,95 \% \mathrm{Cl}^{\prime} \mathrm{s}=-73.50041$ to -31.91053$)$. Lines of best fit was indicated using SMA models.

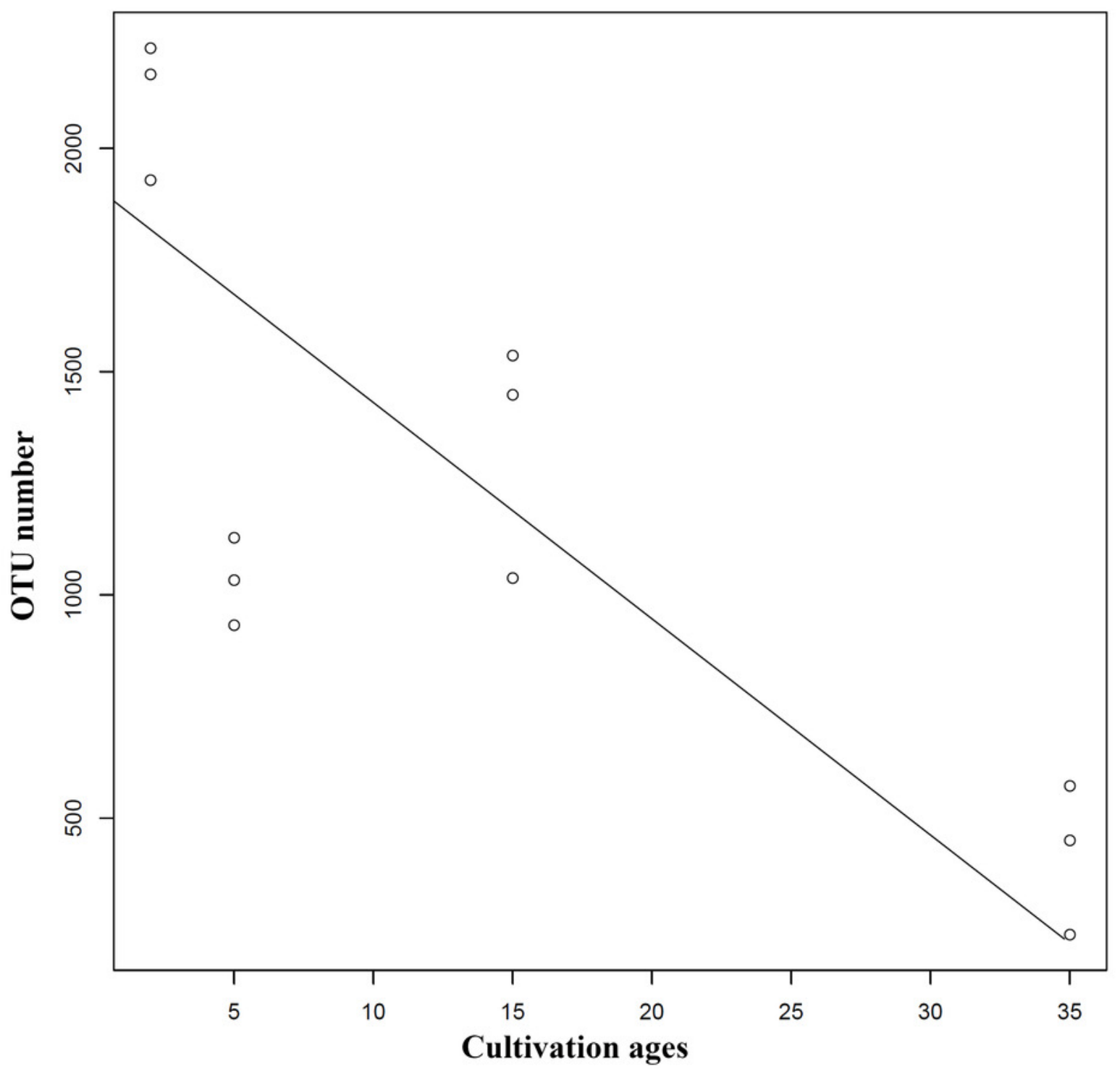


Figure 3

LEfSe comparison of fungi community between C2 and C5

The cladogram represented the hierarchical structure of differently abundant taxa. The taxonomic levels from phylum to family are labeled. C2: farmland cultivated ginseng of 2-yold, C5: farmland cultivated ginseng of 5-y-old.

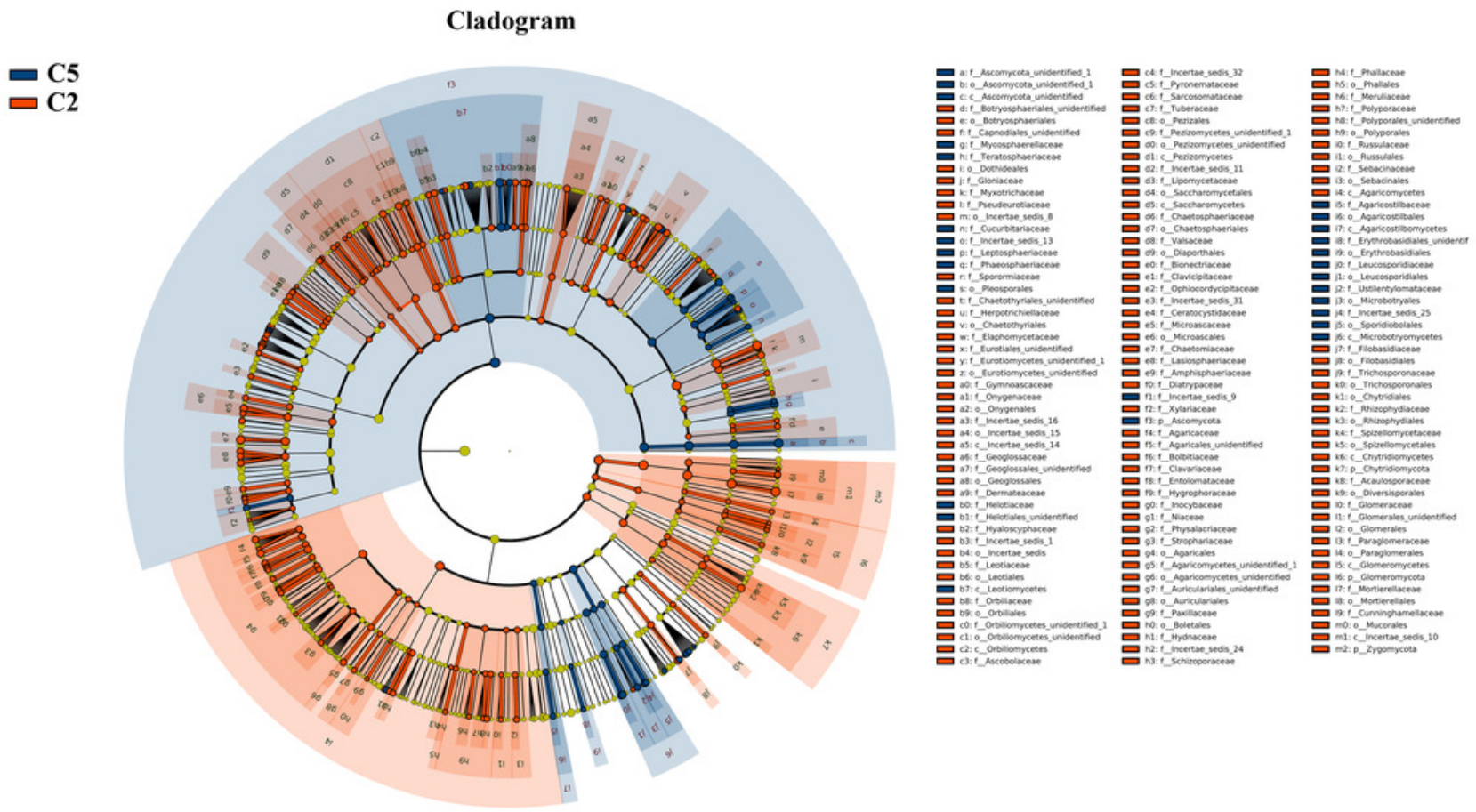


Figure 4

LEfSe comparison of fungi community between W15 and W35

The cladogram represented the hierarchical structure of differently abundant taxa. The taxonomic levels from phylum to family are labeled. W15: understory wild ginseng of 15-yold, W35: understory wild ginseng of 35-y-old. 


\section{Cladogram}
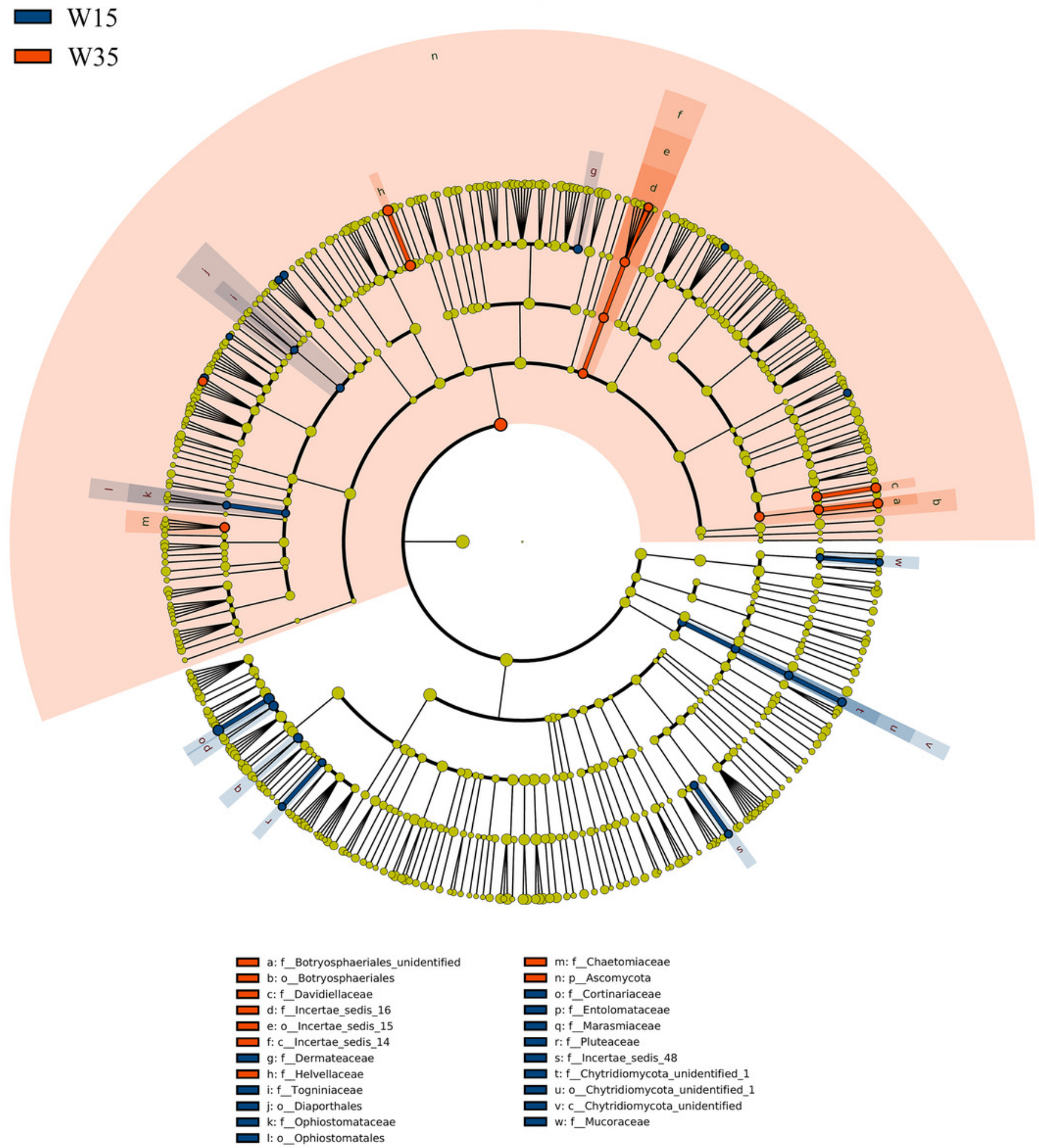


\section{Figure 5}

Non-rhizosphere soil fungal community variation between farmland cultivated ginseng and fallow field.

(A) Venn diagrams illustrating the number of those OTUs that are common between samples of farmland cultivated ginseng soil and fallowed soil. (B) LEfSe comparison of fungi community between F10 and C5. The cladogram represented the hierarchical structure of differently abundant taxa. The taxonomic levels from phylum to family are labeled. C2: farmland cultivated ginseng of 2-y-old, C5: farmland cultivated ginseng of 5-y-old, F10: fallow fields which have been abandoned for ten years after five years of farmland cultivation, W15: understory wild ginseng of 15-y-old, W35: understory wild ginseng of 35-y-old.
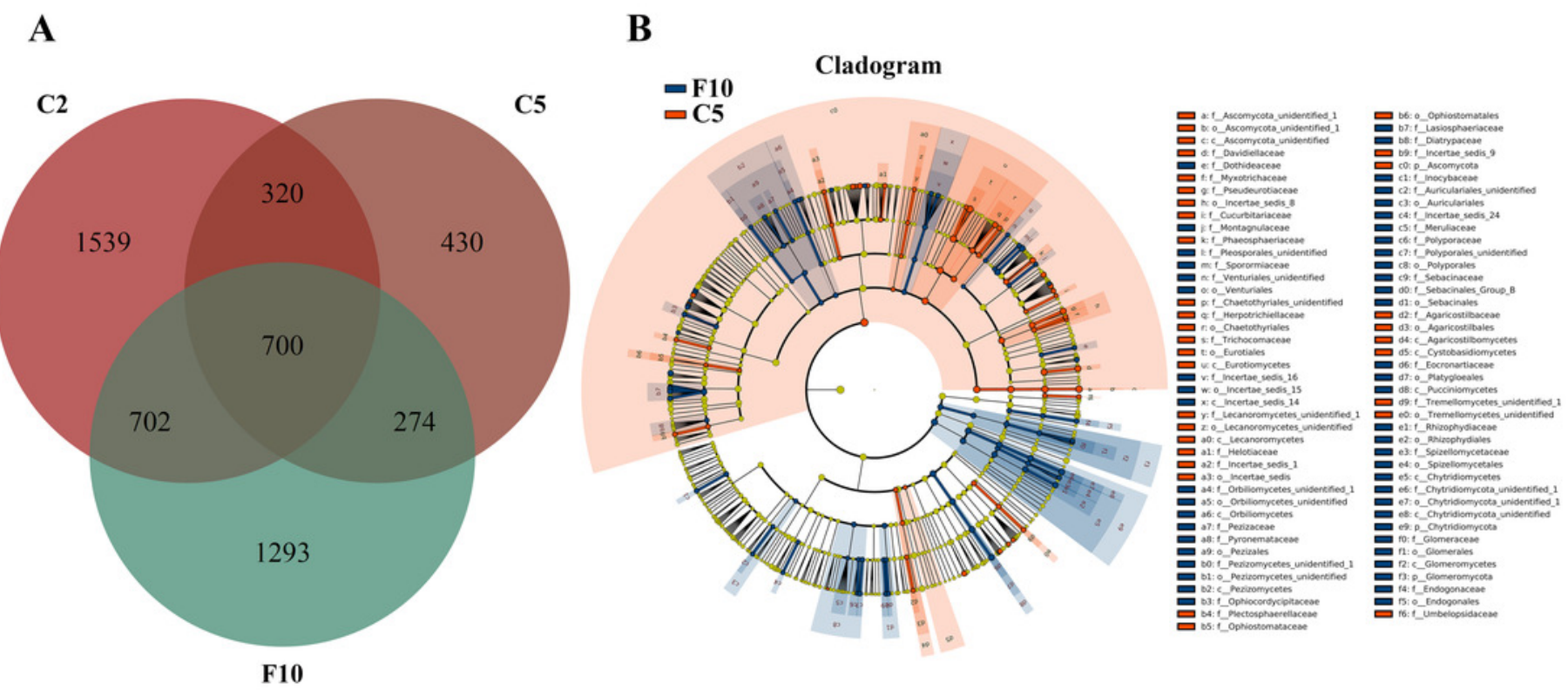

Figure 5 (A) Venn diagrams illustrating the number of those OTUs that are common between samples of farmland cultivated ginseng soil and fallowed soil. (B) LEfSe comparison of fungi community between F10 and C5. Cladogram represented the hierarchical structure of differently abundant taxa. The taxonomic levels from phylum to family are labelled. 


\section{Figure 6}

Relative abundance of fungal functional groups

Putative functional groups were inferred by parsing against the FUNGuild database. C2:

farmland cultivated ginseng of 2-y-old, C5: farmland cultivated ginseng of 5-y-old, F10: fallow fields which have been abandoned for ten years after five years of farmland cultivation, W15: understory wild ginseng of 15-y-old, W35: understory wild ginseng of 35-y-old.

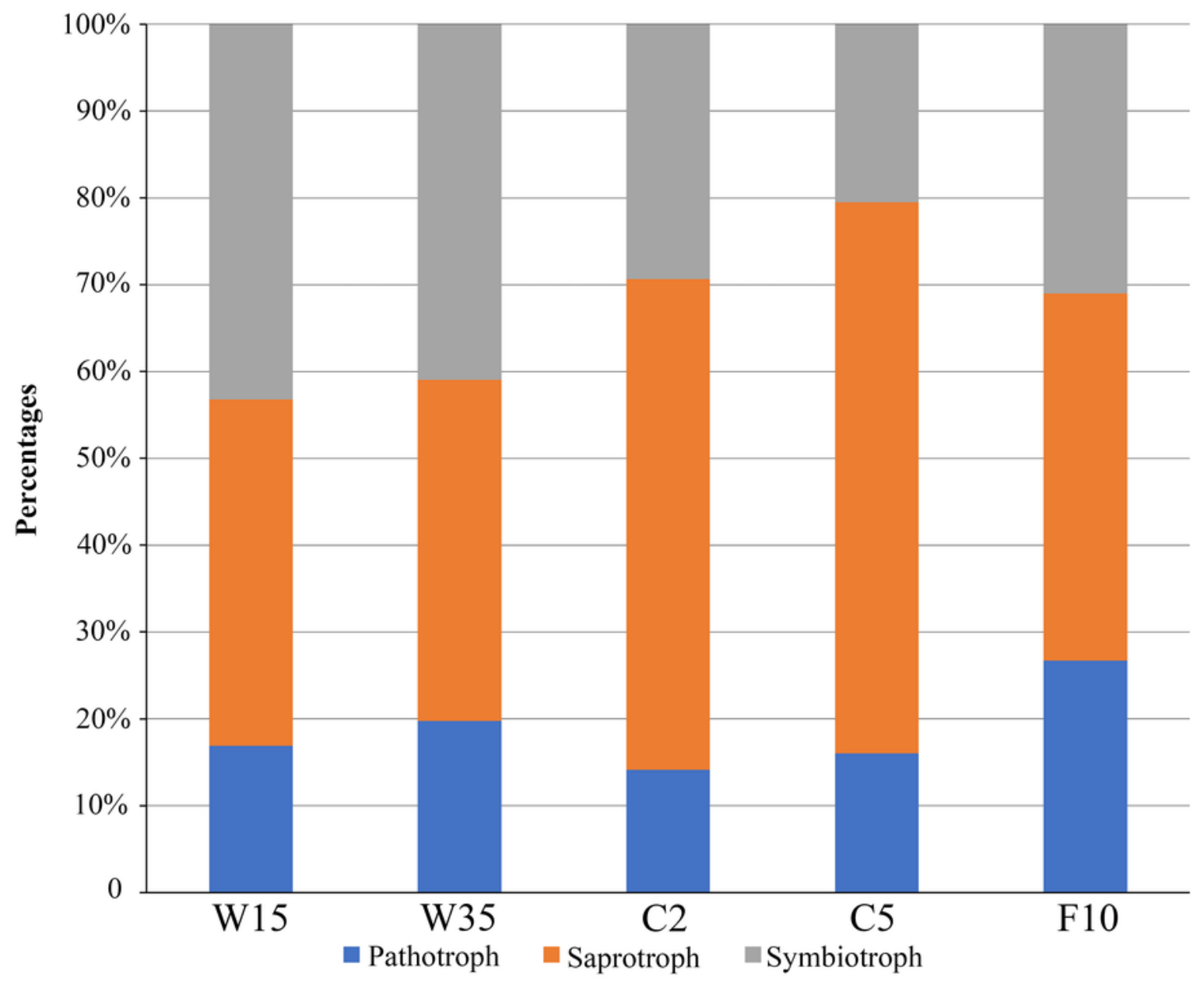




\section{Table 1 (on next page)}

Statistic of alpha diversity indexes in each sample 
1 Table 1 Statistic of alpha diversity indexes in each sample.

2

\begin{tabular}{lllll}
\hline Alpha name & OTUs & PD whole tree & Simpson & Goods coverage \\
\hline W15 & $1341 \pm 265.99$ & $331.4288 \pm 46.1156$ & $0.9688 \pm 0.0054$ & $0.9993 \pm 0.0004$ \\
W35 & $421 \pm 169.01$ & $137.432 \pm 41.1016$ & $0.9759 \pm 0.0097$ & $0.9998 \pm 0.0001$ \\
C2 & $2106.7 \pm 156.7$ & $408.7553 \pm 33.5402$ & $0.9624 \pm 0.0113$ & $0.9973 \pm 0.0004$ \\
C5 & $1031 \pm 98.02$ & $239.2621 \pm 17.9476$ & $0.9523 \pm 0.0182$ & $0.9980 \pm 0.0002$ \\
F10 & $1812.3 \pm 200.9$ & $427.1849 \pm 34.8345$ & $0.9827 \pm 0.0054$ & $0.9976 \pm 0.0003$ \\
\hline
\end{tabular}

3

4 C2: farmland cultivated ginseng of 2-y-old, C5: farmland cultivated ginseng of 5-y-old, F10: fallow fields which 5 have been abandoned for 10 years after 5 years of farmland cultivation, W15: understory wild ginseng of 15-y6 old, W35: understory wild ginseng of 35-y-old. 